\title{
Application of Electron-Beam lonized Discharges to Switches-A Comparison of Experiment with Theory
}

\author{
MARC R. HALLADA, PETER BLETZINGER, SENIOR MEMBER, IEEE, AND WILLIAM F. BAILEY
}

\begin{abstract}
A theoretical investigation of high-pressure discharges ionized by an external electron beam (e-beam) was conducted. Only when secondary emission from the cathode and electron-impact ionization of metastable states were included in the analysis did calculated current-voltage $(I-V)$ characteristics for argon and methane discharges compare well with experimental data. The $I-V$ characteristics obtained reveal a sharp rise in the current at a certain threshold voltage. This threshold voltage and the entire $I-V$ characteristic are shifted to lower voltages when metastable ionization is significant. Below the threshold voltage and at low external ionization source strengths, a region of negative differential conductivity is obtained. In the high-current region, the $I-V$ slope is controlled by the secondary emission coefficient. The additional cathode sheath ionization from secondary emission and ionization from metastable states significantly reduces the discharge voltage. This important effect can be used to reduce $e$-beam switch losses and increase lifetime through judicious gas mixture selection and proper cathode conditioning.
\end{abstract}

\section{INTRODUCTION}

$\mathrm{H}^{\prime}$ IGH-PRESSURE DISCHARGES ionized by the external electron beam ( $e$-beam) offer unique advantages for use as high-power switches. They are capable of switching, both on and off, large currents at high voltages. Initial experiments [1], [2] using very high $e$-beam current densities, have proven the feasibility of this concept. Recent experiments [3], which used very low $e$-beam current densities, achieved a much higher current gain, i.e., the ratio of the discharge current switched to the $e$-beam current. In these experiments, discharge current densities of more than $1 \mathrm{~A} / \mathrm{cm}^{2}$ have been achieved and current densities of $10 \mathrm{~A} / \mathrm{cm}^{2}$ are considered to be obtainable. The $e$-beam current density required to control the switch is low, ranging from 1.5 to $50 \mathrm{~mA} / \mathrm{cm}^{2}$. Thus using a moderate power electron gun (e-gun), "on" operation time of $10^{-5}-10^{-4} \mathrm{~s}$ are achieved without being limited by overheating of the foil which separates the $e$-gun vacuum and the discharge gas.

The repetition rate of these switches is limited only by the cooling characteristics of the gas volume. It is, therefore, desirable to minimize the heating of the discharge gas, and consequently the voltage drop across the discharge. Three approaches to minimizing this discharge voltage drop have been considered. The first approach, increasing the $e$-beam

Manuscript received March 30, 1982; revised July 2, 1982.

M. R. Hallada and W. F. Bailey are with the Air Force Institute of Technology, Wright-Patterson AFB, OH 45433.

P. Bletzinger is with the Air Force Wright Aeronautical Laboratories, Wright-Patterson AFB, OH 45433. current density, has already been shown in [1] to result in an undesirable reduction in the current gain of the switch. Another approach pursued in [2], [3] is based upon using gases or gas mixtures with very high drift velocity. The third method optimizes the additional ionization in the cathode sheath which has been predicted by [4], [5] and measured in some detail in [3].

In order to improve $e$-beam switch design through judicious gas mixture selection and enhancement of cathode sheath ionization processes, a better theoretical understanding of these discharges is required. The theory of $e$-beam ionized discharges has been treated by numerous authors and the effect of cathode sheaths has been investigated in [4], [5]. A particularly clear treatment was presented by Lowke and Davies [6]. They investigated externally ionized discharges at the very low gas pressure and electron density usually associated with charged particle counters. They also considered the intermediate gas pressure and electron density case for an $e$-beam ionized discharge. This paper, based on their theoretical development, analyzes an externally ionized discharge at higher current densities and considers the important effects of secondary emission by ion bombardment of the cathode and ionization via metastable states. These theoretical results are compared to experimental measurements in argon and methane and their implications for $e$-beam controlled switches are discussed.

\section{BASIC EQUATIONS AND Assumptions}

A one-dimensional geometry is adopted with the cathode located at the position $x=0$ and the anode at $x=d$. The positive direction for the electric field, current densities, and spatial coordinate are thus defined to be from cathode to anode. The $e$-beam source strength, $S$ (electron-ion pairs $\mathrm{cm}^{-3} \cdot \mathrm{s}^{-1}$ ), is assumed to be spatially uniform and independent of the voltage applied to the plane-parallel electrodes.

Space-charge effects in these discharges are significant. Consequently, particular attention has been directed toward analyzing the electrode sheath regions. In such an analysis, it is necessary to consider the detailed interactions occurring within the plasma. Experimental data or theoretical values based upon a numerical solution of the collisional Boltzmann equation are used to establish the reaction rates (ionization, excitation, and recombination) and transport properties (mean energy, drift velocity, and diffusion coefficient). Use of these rates assumes that the discharge is in equilibrium with the local 
value of the sustaining electric field. The validity of this assumption may be questioned in the electrode regions where spatial variations in the electric field are significant; however, the major features of the solutions that are obtained are unlikely to be significantly in error [6].

Based upon an assumed quasi-equilibrium, the gas discharge was analyzed using a set of 4 coupled first-order differential equations. These equations are: the continuity equations for electrons and positive ions, the electron flux equation, and Poisson's equation. A simultaneous solution of this set of equations, subject to the appropriate boundary conditions, permitted the determination of the spatial variation of discharge quantities such as electron and ion densities, the electric field, and the electron and ion fluxes.

The continuity equation for electrons is

$$
\frac{\partial n_{e}}{\partial t}=S+\alpha\left|W_{e}\right| n_{e}+R_{i m} n_{e} n_{m}-R_{r} n_{e} n_{+}-\frac{\partial j_{e}}{\partial x}
$$

where $n_{e}$ is the electron density, $n_{+}$is the ion density, $t$ the time, $\alpha$ is Townsend's first ionization coefficient, $W_{e}$ is the electron drift velocity, $R_{i m}$ is the metastable ionization coefficient, $n_{m}$ is the number density of metastable neutrals, $R_{r}$ is the recombination coefficient, $j_{e}$ is the electron flux, and $x$ is the distance from the cathode. Similarly, the continuity equation for positive ions is

$$
\frac{\partial n_{+}}{\partial t}=S+\alpha\left|W_{e}\right| n_{e}+R_{i m} n_{e} n_{m}-R_{r} n_{-} n_{+}-\frac{\partial j_{+}}{\partial x} .
$$

In the steady state, the time derivatives vanish, and the result is

$$
\frac{\partial j_{e}}{\partial x}=\frac{\partial j_{+}}{\partial x}=S+\alpha\left|W_{e}\right| n_{e}+R_{i m} n_{e} n_{m}-R_{r} n_{e} n_{+} .
$$

The electron flux is given by

$$
j_{e}=n_{e} W_{e}-D_{e} \frac{\partial n_{e}}{\partial x}
$$

where $D_{e}$ is the electron diffusion coefficient. The companion equation for ion flux simplifies to

$$
j_{+}=n_{+} W_{+}=n_{+} k_{+} E
$$

where we have discarded the ion diffusion current which is very much smaller than the ion drift current. Here $W_{+}$is the ion drift velocity, $k_{+}$is the ion mobility, and $E$ is the electric field. The potential $V(x)$ is obtained from Poisson's equation

$$
\frac{\partial^{2} V}{\partial x^{2}}=\frac{-e}{\epsilon_{0}}\left(n_{+}-n_{e}\right)
$$

where $e$ is the electronic charge and $\epsilon_{0}$ is the permittivity. The electric field is obtained from the potential

$$
E=-\frac{\partial V}{\partial x}
$$

In summary, the steady-state behavior of an externally sustained gas discharge may be described by a set of 4 coupled differential equations with the 4 independent variables $j_{e}$, $n_{e}, n_{+}$, and $E$

$$
\begin{aligned}
& \frac{\partial j_{e}}{\partial x}=S+\alpha\left|W_{e}\right| n_{e}+R_{i m} n_{e} n_{m}-R_{r} n_{e} n_{+} \\
& \frac{\partial j_{+}}{\partial x}=S+\alpha\left|W_{e}\right| n_{e}+R_{i m} n_{e} n_{m}-R_{r} n_{e} n_{+} \\
& \frac{\partial n_{e}}{\partial x}=\frac{n_{e} W_{e}-j_{e}}{D_{e}} \\
& \frac{\partial E}{\partial x}=\frac{e}{\epsilon_{0}}\left(n_{+}-n_{e}\right) .
\end{aligned}
$$

The ion flux $j_{+}$is calculated from $n_{+}$and $E$ using (5).

At the high currents and source strengths of interest for switching applications, a positive column region is established in the interelectrode gap. In this region, the electric-field strength is approximately constant. Thus $\partial E / \partial x \simeq 0$ and $n_{+} \simeq n_{e}$ from (8d). Spatial gradients in the electron and ion number densities and the electron flux are also very small in the positive column. Thus $\partial n_{e} / \partial x \simeq 0$ and $\partial j_{e} / \partial x \simeq 0$. Therefore, in the positive column, $j_{e}=n_{e} W_{e}$ from (8c). In the weak positive column field, $e$-beam ionization dominates. Therefore, $n_{e} \simeq\left(S / R_{r}\right)^{1 / 2}$ from (8a). In addition, the total current in the positive column $J$ is carried primarily by the electrons so that $j_{e} \simeq J / e$. This fact is then used to determine the value of $E$ in the positive column consistent with

$$
W=\frac{J}{n_{e} e} .
$$

Therefore, all 4 of the independent variables can be specified in the positive column region

$$
\begin{aligned}
& j_{e}=J / e \\
& n_{e}=n_{+}=\left(S / R_{r}\right)^{1 / 2}
\end{aligned}
$$

and

$$
E \text { from } \frac{J}{n_{e} e} \text {. }
$$

The 4 differential equations (8) are then numerically integrated from the positive column region to each of the electrodes where the appropriate boundary conditions, developed in the next section, are imposed. These coupled equations are extremely sensitive to deviations from charge neutrality and possess widely separated eigenvalues. To ensure numerical stability we have utilized the multistep implicit scheme proposed by Gear [7].

\section{SPECIFICATION OF BOUNDARY CONDITIONS}

The boundary conditions at the electrodes were determined from the physical requirements on the ratio of electron to ion fluxes at the electrodes. The secondary emission coefficient $\gamma$ is defined as the ratio of the liberated electron and incident ion fluxes at the cathode. In this analysis, coefficients ranging from 0.001 to 0.050 have been considered. Expressing the electron flux at the cathode in terms of the secondary emission coefficient and the positive ion flux or total current $J$, the cathode boundary condition is

$$
j_{e}=\gamma j_{+}=\frac{\gamma}{\gamma-1} \frac{J}{e}
$$


where we have imposed the current continuity requirement that $J / e=j_{e}-j_{+}$.

In specifying the boundary condition at the anode, 2 different cases must be considered. In the first case, the electric field is positive at the anode. Positive ions are therefore driven toward the anode and yield secondary electrons on impact. In this case, the boundary condition at the anode is identical to that at the cathode, namely (11). Conversely, if the electric field is negative in the anode region, electrons are driven into the anode producing an ion flux defined by (12)

$$
j_{+}=\eta j e
$$

where $\eta$ is the ion secondary emission coefficient. This coefficient is extremely small; thus we can approximate the boundary condition at the anode for $E>0$ as $j_{+}=0$. Using this approximation, (5) implies $n_{+}=0$ at the anode for $E \neq 0$.

Subject to these boundary conditions, the coupled set of differential equations (8) were integrated. In the following sections, the resulting distributions of the number density of positive ions and electrons and the spatial variation of the discharge voltage are presented. These macroscopic distributions are then used to generate theoretical current-voltage characteristics for comparison with experimental data.

\section{Distribution of Electron and Ion Densities in THE High-CurRent CASE}

With switching applications in mind, we will restrict our theoretical investigation to current densities above $10 \mathrm{~mA} / \mathrm{cm}^{2}$ where the increased electric field in the cathode sheath causes significant Townsend ionization. As a point of reference, however, Fig. 1 shows the electron and ion densities and discharge voltage calculated for a current below this ionization threshold. In this case the positive space charge in front of the cathode produces a large electric field ( $4 \mathrm{kV} / \mathrm{cm}$ max), but it is not sufficient to cause significant Townsend ionization. When the discharge current is increased and the ionization threshold exceeded (Fig. 2), significant Townsend ionization in the cathode, sheath occurs and a peak in the positive ion density appears. The peak arises because the Townsend ionization rate, which is proportional to $n_{e} \exp (-(A / E))$, is small near the cathode, where the electron density is low. This rate increases as the electron density increases in the sheath region and then decreases close to the beginning of the positive column where the electric field is small. Fig. 2 also shows the influence of increasing source strength. The cathode sheath region is compressed. The cathode fall and the voltage drop in the positive column are decreased and the electric field at the cathode is also decreased. The peak positive ion density, however, changes little in magnitude.

An important effect mentioned by Lowke and Davies but not considered in their calculations (see, however, [4] , [5]), is secondary electron emission from the cathode by ion bombardment. Depending on ion energy, cathode material, and surface condition, the secondary emission coefficient $\gamma$ can vary over a wide range of values.

Fig. 3 compares the calculated distribution of electron and

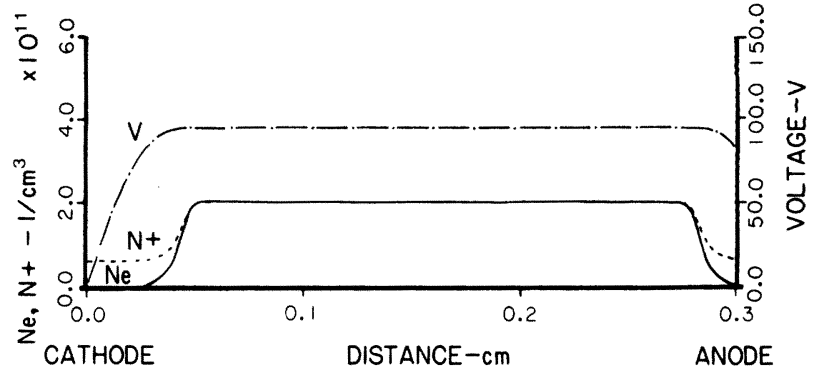

Fig. 1. Calculated distribution of electron and ion densities and discharge voltage along discharge axis below threshold current for ionization in cathode sheath. Argon 240 torr, $S=3.6 \times 10^{16} \mathrm{~cm}^{-3}$ / $\mathrm{s}^{-1}, J=0.254 \mathrm{~mA} / \mathrm{cm}^{2}$.
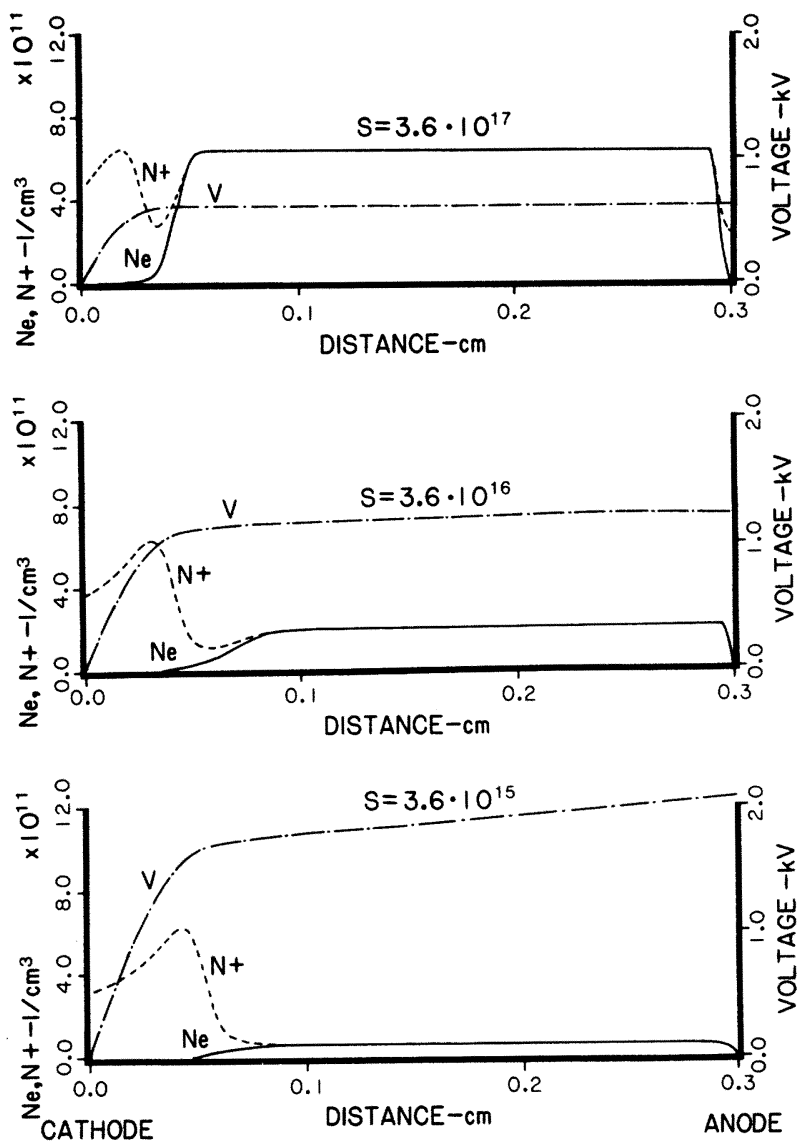

Fig. 2. Calculated distribution of electron and ion densities and discharge voltage along discharge axis for different source strengths $S\left(\mathrm{~cm}^{-3} \cdot \mathrm{s}^{-1}\right)$. Argon 240 torr, $J=15 \mathrm{~mA} / \mathrm{cm}^{2}$.

ion densities in argon at 240 torr for 2 values of $\gamma(0.0$ and $0.02)$. Significant changes occur. The discharge voltage drops by a factor of 2 , revealing that switch losses can be decreased by proper conditioning and selection of cathode material. Similar to the previous case of increasing source strength, Fig. 2, the cathode sheath is compressed as $\gamma$ increases.

For discharges in which electrons and ions are solely produced by Townsend ionization, a linear relationship between the cathode fall and the natural logarithm of $\gamma$ is predicted [8]. As shown in Fig. 4, this is nearly the case for low source strengths. However, as the source strength increases, the source ionization exceeds the contribution from Townsend ionization, resulting in a departure from linearity for small values 

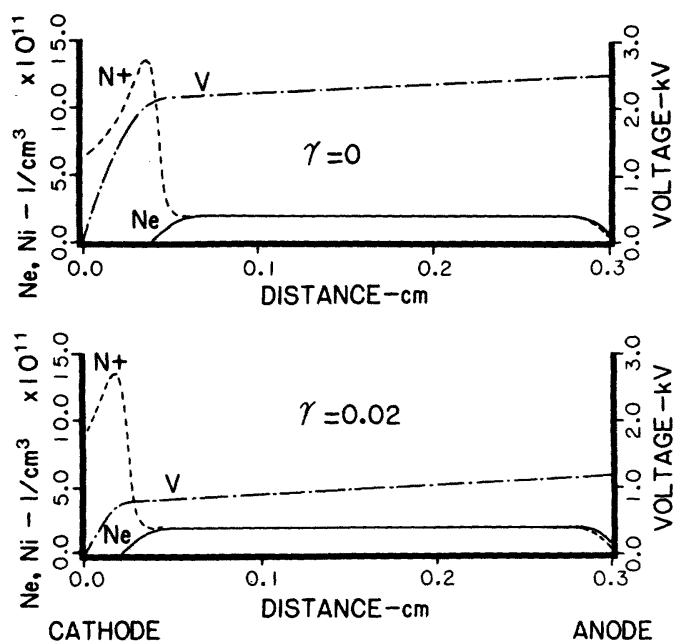

Fig. 3. Calculated distribution of electron and ion densities and discharge voltage along discharge axis with the secondary emission coefficient as parameter. Argon 240 torr, $S=3.6 \times 10^{-16} \mathrm{~cm}^{-3} /$ $\mathrm{s}^{-1}, J=48 \mathrm{~mA} / \mathrm{cm}^{2}$.

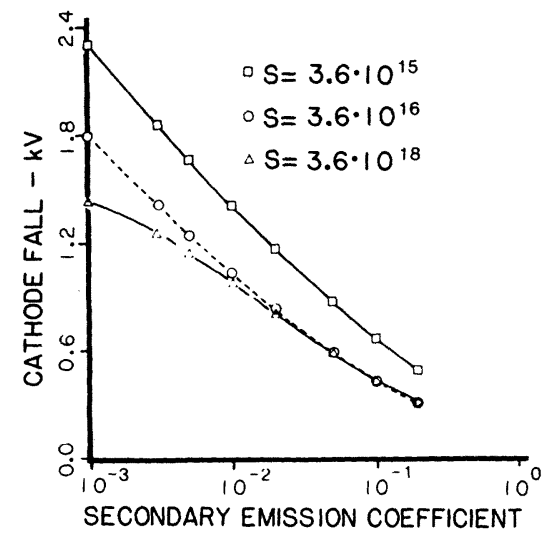

Fig. 4. Calculated cathode fall versus secondary emission coefficient for various values of source strengths $S \mathrm{~cm}^{-3} \cdot \mathrm{s}^{-1}$. Argon 760 torr, $J=500 \mathrm{~mA} / \mathrm{cm}^{2}$.

of $\gamma$. This lowering of the cathode fall with increasing source strength saturates at large values of $\gamma$.

\section{COMPUTED AND EXPERIMENTAL $I-V$ Characteristics}

Current-voltage characteristics, for a given source strength and electrode separation, were constructed from a series of calculations using the current density as the independent variable. Initially these calculations were performed for a gas pressure of 760 torr, an electrode separation of $0.3 \mathrm{~cm}$, a secondary emission coefficient of 0.02 , and considered only Townsend ionization from the ground electronic state. Figs. 5 and 6 show the results obtained for argon. At low-current densities, almost the entire voltage drop in the discharge occurs in the cathode sheath and the discharge current density increases relatively slowly with increasing voltage (region $A B$, Fig. 6). At a particular threshold or "ignition" voltage, there is a sharp increase in the slope of the $I-V$ curve. Beyond this ignition voltage, the discharge current density increases rapidly (region $C D$ ). This sharp rise in the current density is due to the rapid increase of Townsend ionization in the cathode sheath. In this current density/voltage regime, the current

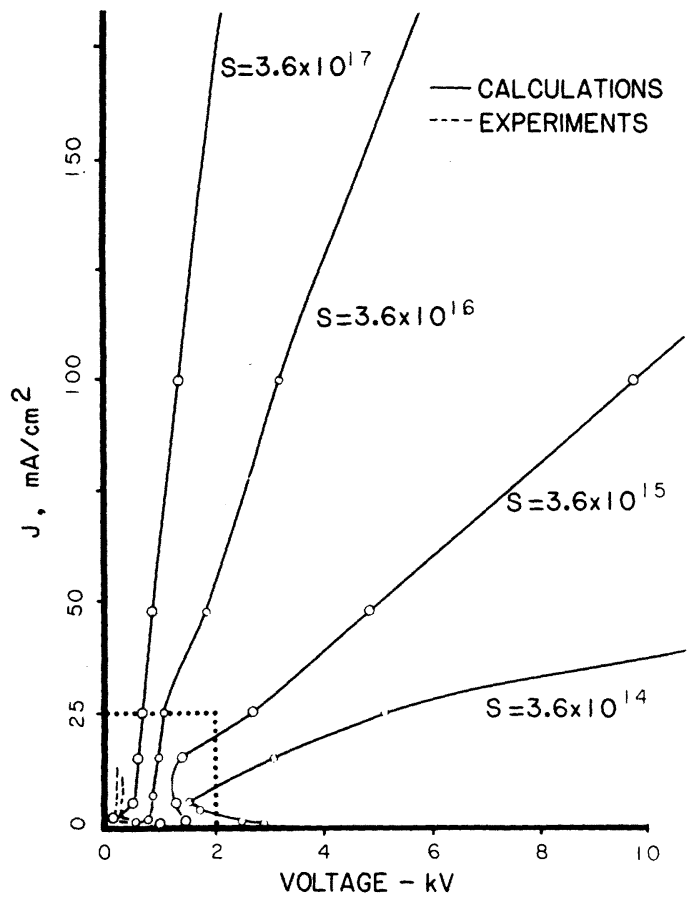

Fig. 5. $I-V$ characteristic calculated for an argon discharge for various values of source strengths $S \mathrm{~cm}^{-3} \cdot \mathrm{s}^{-1}(760$ torr $=0.02, d=0.3 \mathrm{~cm}$, no metastable ionization)

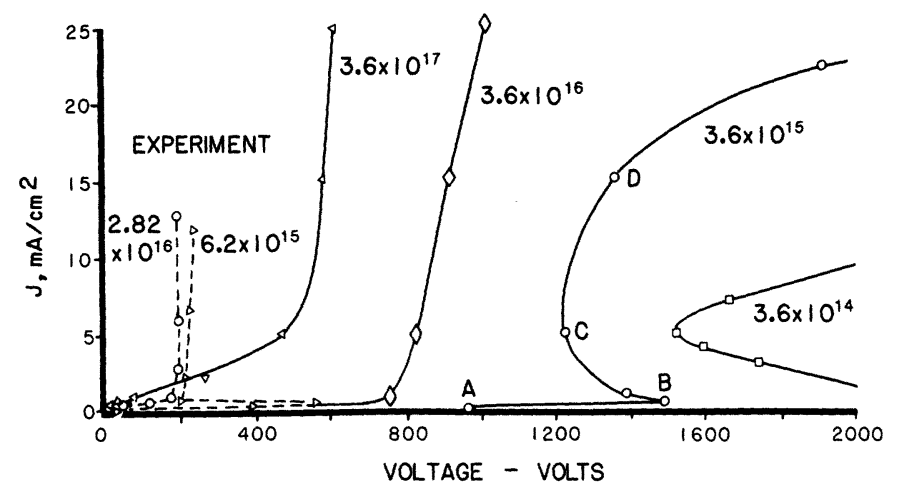

Fig. 6. I-V characteristic calculated for an argon discharge for various values of source strengths $S \mathrm{~cm}^{-3} \cdot \mathrm{s}^{-1}$ (expansion of low current and voltage region of Fig. 5.

density is then determined primarily by the mobility and density of electrons in the plasma region and is approximately proportional to the total discharge voltage.

The transition from the low to high conductivity regions occurs smoothly for large external ionization source strengths. However, at lower source strengths, regions of negative differential conductivity occur, as in region $B C$ (Fig. 6). In this $I-V$ regime, the effective resistance is negative as the discharge reconfigures itself into a more efficient operating mode. This behavior can be compared to the transition from a subnormal to a normal glow discharge. These regimes of negative differential conductivity were also observed in similar calculations by Velikhov et al. [4] and Zakharov et al. [5], for $\mathrm{N}_{2}$ discharges. Although Zakharov [5] maintained that this region was not physically observable, Averin et al. [9] reported that by using a large enough external (load) impedance, they were able to experimentally map out this transition region. The 


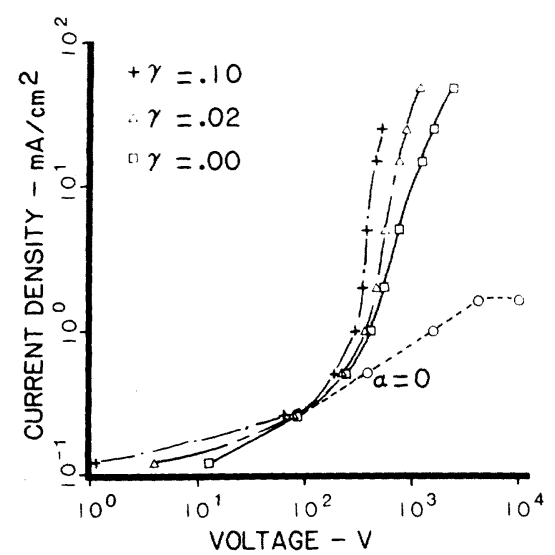

Fig. 7. Calculated $I-V$ characteristics for 3 secondary emission coefficients (argon 240 torr, $S=3.6 \times 10^{16} \mathrm{~cm}^{-3} / \mathrm{s}^{-1}$ ). For reference, a curve neglecting Townsend ionization $(\alpha=0)$ is shown also.

experimental results from [3] are also shown in Fig. 6. The negative differential conductivity regime was observed in this experiment, but only as an unstable transition from one $I-V$ region to another, even when using a current source (corresponding to an infinte load resistance). At larger source strengths, where the cathode sheath field and sheath thickness are reduced (see Fig. 2), this unstable region disappears and the transition from external ionization control of the sheath to a combination of Townsend and external ionization occurs smoothly.

Three discrepancies are apparent between the theoretical and experimental characteristics displayed in Fig. 6: the experimental curves have a much steeper slope, the separation between the experimental $I-V$ characteristics is smaller, and the discharge voltage is lower. The slope in the high conductivity region is controlled by the secondary emission coefficient, Fig. 7. This slope increases with increasing $\gamma$ as is also shown in Fig. 7. Based on this identification and scaling, the experimental data suggest that a much higher value of $\gamma$ than that associated with the ground state is effective. One is led to consider the influence of excited, or more specifically, metastable states. Secondary emission coefficients as large as 0.50 have been reported for argon metastables [10]. In addition, there is spectroscopic evidence that significant concentrations of metastable argon atoms are present in this type of discharge [3]. Theoretical $I-V$ characteristics with slopes similar to those observed experimentally would therefore result from the much larger and more realistic value of $\gamma$ associated with the metastable argon state.

The presence of metastable argon atoms may also explain both the smaller separation and the lower voltages. Here it is not the increased value of $\gamma$ that is important, but Townsend ionization via metastable states. The lower ionization potential of metastable states will significantly reduce the required cathode fall potential and soften the sensitivity of the cathode fall to variations in source strength.

Using a theoretical cross section calculated by Flannery [11] and assuming a Maxwellian electron velocity distribution, a metastable ionization rate was obtained. Accounting for this alternate ionization channel, an $I-V$ curve was calculated for a source strength of $3.6 \times 10^{16}$ in a 760-torr argon discharge

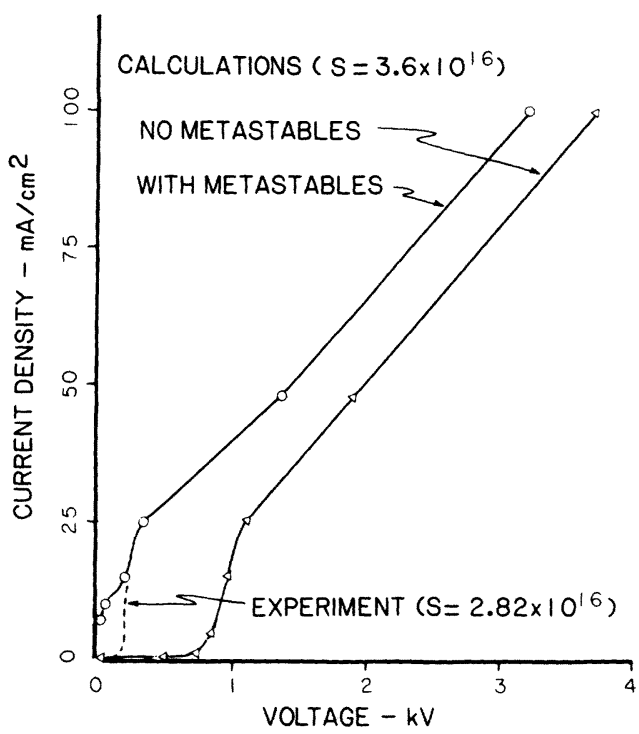

Fig. 8. Experimental and calculated $I-V$ characteristics for an argon discharge (same parameters as Fig. 5, but with metastable ionization). Experimental source strength $2.81 \cdot 10^{16} \mathrm{~cm}^{-3} \cdot \mathrm{s}^{-1}$, source strength for calculated curve $3.6 \times 10^{16} \mathrm{~cm}^{-3} \cdot \mathrm{s}^{-1}$.

(Fig. 8). The value of the secondary emission coefficient was maintained at 0.02. An experimental curve from [3] with a similar source strength and electrode gap is included for comparison. Although the metastable calculation was not carried to current densities below $7 \mathrm{~mA} / \mathrm{cm}^{2}$, the experimental and calculated curves are now in reasonable agreement where they overlap. Thus the addition of metastable ionization shifts the $I-V$ characteristic to lower discharge voltages, while leaving the slope of the high conductivity portion of the characteristic relatively unchanged.

Further confirmation of the presence and importance of argon metastables is obtained by analyzing the electric field in the positive column. Assuming that Townsend ionization from the ground state is negligible, the number density of electrons in the positive column is obtained from the electron continuity equation (8a). Recalling that $\partial j_{e} / \partial x \simeq 0$ in the positive column, a quadratic equation for $n_{e}$ is obtained

$$
S-R_{r} n_{e}^{2}+R_{i m} n_{e} n_{m}=0
$$

with the solution

$$
n_{e}=\frac{R_{i m} n_{m}+\sqrt{\left(R_{i m} n_{m}\right)^{2}+4 S R_{r}}}{2 R_{r}} .
$$

When metastable ionization is significant, the electron number density is increased resulting in a lowering of the electric field in the positive column for a fixed current. Using the same metastable ionization rate and the same source strength as previously, $R_{r}=8.81 \times 10^{-7} \mathrm{~cm}^{3} \cdot \mathrm{s}^{-1}$, an electron density of $4 \times 10^{12} \mathrm{~cm}^{-3}$, and an $E / N$ of 0.004 Townsend $\left(10^{-17} \mathrm{~V}\right.$ $\mathrm{cm}^{2}$ ) are obtained in the positive column. This compares favorably with the experimental value of 0.01 Townsend measured in [3]. Thus using relatively simple assumptions, the very small electric fields experimentally observed in the positive column can be explained by the presence of metastables and the resulting two-step ionization.

Methane has been suggested as a gas particularly well suited 


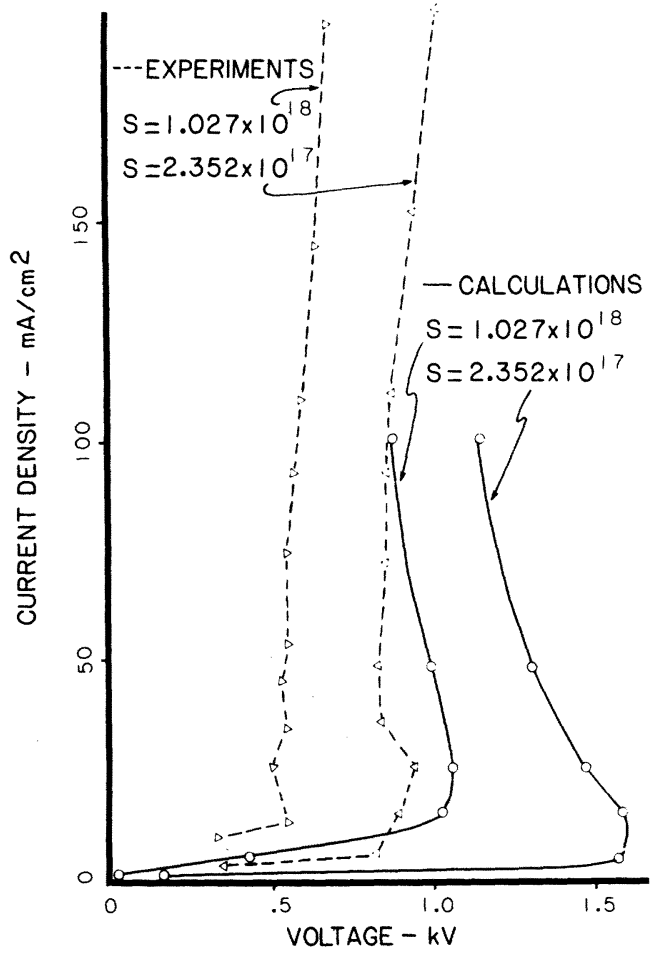

Fig. 9. Experimental and calculated $I-V$ characteristics for the methane discharge $(760$ torr $=0.02, d=2.2 \mathrm{~cm})$ with the indicated source strength $S\left(\mathrm{~cm}^{-3} \cdot \mathrm{s}^{-1}\right)$.

for an $e$-beam controlled switch application because of its very high drift velocity and high holdoff field strength. Using the same computational methods, $I-V$ curves were calculated for methane discharges at 760 torr and compared in Fig. 9 with the experimental results of [3]. Although the calculated curves are shifted to higher discharge voltages, the spacing between different source strength curves is nearly the same for both sets of curves. In addition, both experimental and theoretical curves consistently exhibit transition regions of negative differential conductivity. In these regions, the experimental curves are somewhat distorted by the influence of the external impedance. In addition, the determination of experimental source strengths has limited accuracy. These factors contribute to some of the discrepancies between experimental and theoretical results. The extended negative differential conductivity region in the theoretical curves is sensitive to the assumed value of the secondary emission coefficient of 2 percent, which may be too large for methane. In contrast with argon, metastable ionization in methane was not included since data was lacking on metastable states and their ionization cross sections.

Even though in methane the effects of the ionization in the cathode sheath are less dramatic, at high-current densities they are still very important. In Fig. 10 experimental $I-V$ curves are compared to a computed $I-V$ curve, based simply on the solution of the continuity equation. Here, the cathode fall voltage and its dependence on discharge current densities were extracted from the experimental results. As can be seen from Fig. 10, at the highest current densities before the flat region of the $I-V$ curve (approx. $1.1 \mathrm{~A} / \mathrm{cm}^{2}$ ), which is the preferred operating region for such a discharge, the difference between the (simple) theoretical and measured characteristics is about

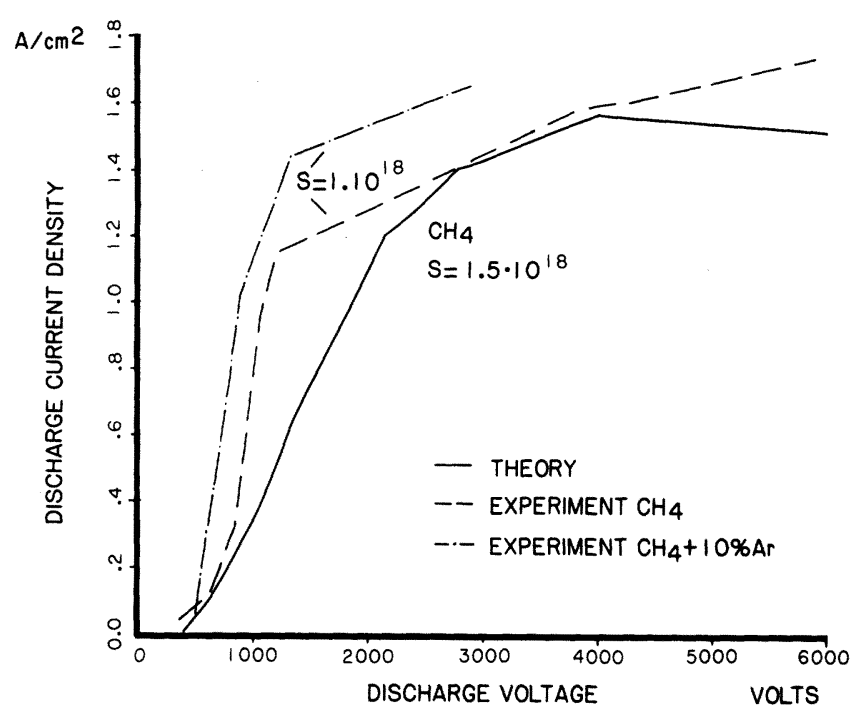

Fig. 10. Comparison of experimental characteristic of methane and methane/argon discharge with simple continuity equation model not considering cathode sheath effects. (Gas pressures all 760 torr, source strength $S\left(\mathrm{~cm}^{-3} \cdot \mathrm{s}^{-1}\right)$ as indicated).

$1 \mathrm{kV}$. At the large currents projected for such a device, this would add considerably to the power losses in the switch. Moreover, when in the experiment argon was added to the gas, a further reduction in discharge voltage was observed. The reduction of the discharge voltage on addition of argon is most likely due to the effect of metastable ionization in the argon. Based upon the magnitude of this voltage shift, metastable effects may have already been active in the pure methane discharge. Since the slope of the experimental $I-V$ curve in this region did not change, a change in the effective secondary emission coefficient upon addition of argon is improbable. The increase in current density in the flat portion of the $I-V$ curve is probably due to a higher drift velocity of the methane-argon mixture at the $E / N$ value used compared to pure methane [12].

\section{CONCLUSIONS}

The very nonlinear $I-V$ characteristics of $e$-beam ionized argon and methane discharges can be understood in terms of cathode sheath effects and the associated additional Townsend ionization generated in the sheath region. As in the conventional cathode fall of a self-sustained discharge, the cathode fall of an $e$-beam ionized discharge is strongly influenced by the cathode secondary emission coefficient. Unlike the conventional discharge, the cathode fall of an $e$-beam discharge exhibits a strong dependence on the discharge current and is sensitive to the $e$-beam source strength.

The importance of Townsend ionization in the cathode sheath decreases when the external source strength is increased. However, if the current gain of $e$-beam controlled switches is to be in the range $10^{2}-10^{3}$, the $e$-beam source strength must be limited to values less than $10^{19}$ electron pairs $\mathrm{cm}^{-3} \cdot \mathrm{s}^{-1}$. The same limit is imposed by requirements for "on" times of $10^{-5}-10^{-4} \mathrm{~s}$ and high duty cycles. Consequently, in the parameter range of interest for switching applications, the cathode sheath ionization must indeed by considered and optimized.

Optimization of ionization in the cathode sheath can lead to 
a substantial reduction in switch losses associated with the cathode fall voltage. For the case of atmospheric pressure argon, this reduction can amount to a factor of 20 ; in methane-argon mixtures, a 50-percent reduction is achieved. The reduction is enhanced by selecting gases or gas mixtures with large cross sections for ionization via a metastable state, and large secondary emission coefficients, (e.g., argon and methane/argon mixtures). A further benefit is obtained from conditioning the cathode surface using selected cathode materials.

\section{REFERENCES}

[1] B. M. Koval'chuk, V. V. Kremnev, G. A. Mesyats, and Yu F. Potalitsyn, "The injection thyratron-A completely controlled ion device," Sov. Radio. Eng. El. Phys., vol. 21, p. 1513, 1976.

[2] R. O. Hunter, "Electron beam controlled switching," in Proc. Int. Pulse Power Conf., Paper IC8, Lubbock, TX, 1976.

[3] P. Bletzinger, "Electron beam switching experiments in the high current gain regime," in Proc. 3rd Int. Pulse Power Conf., Paper 5.4, Albuquerque, N.M.

[4] E. P. Velikhov, V. D. Pis'mennyl, and A. D. Rakhimov, "The nonself-sustaining gas discharge for exciting continuous-wave gas lasers," Sov. Phys. - Usp., vol. 20, p. 586, 1977.

[5] V. V. Zakharov, A. A. Karpikov, and E. V. Chekhonov, "Gas discharge in nitrogen with steady-state external ionization," Sov. Phys. -Tech. Phys., vol. 21, p. 1074, 1976.

[6] J. J. Lowke and D. K. Davies, "Properties of electric discharges sustained by a uniform source of ionization," J. Appl. Phys., vol. 48 , p. 4991, 1977.

[7] A. C. Hindmarsh, "Gear: Ordinary differential equation system solver," Lawrence Livermore Laboratory, UCID-30001, rev. 3, Dec. 1974.

[8] A. von Engel, Ionized Gases. Oxford, England: Clarendon Press, 1955 , chap. 8.

[9] A. P. Averin, Ye. V. Glotov, V. A. Danilychev, V. N. Koterov, A. M. Soroka, and V. I. Yugov, "Negative differential conductivity of electron-ionization discharge in nitrogen," Pism, Zhurn. Tekhn. Fiz., vol. 6, p. 405, 1980.

[10] - chap. 3 .

[11] D. Ton-That and M. R. Flannery, "Cross-sections for ionization of metastable rare-gas atoms ( $\left.\mathrm{Ne}^{*}, \mathrm{Ar}^{*}, \mathrm{Kr}^{*}, \mathrm{Xe}^{*}\right)$ and of metastable $\mathrm{N}_{2}{ }^{*}, \mathrm{CO}^{*}$ molecules by electron impact," Phys. Rev., vol. A15, p. 517, 1977.

[12] L. Foreman, P. Kleban, L. D. Schmidt, and H. T. Davis, "Drift velocities of electrons in methane-inert-gas-mixtures," Phys. Rev. $A$, vol. 23, p. 1553, 1981.

\title{
Performance Predictions for Electron-Beam Controlled On/Off Switches
}

\author{
LAWRENCE E. KLINE, SENIOR MEMBER, IEEE
}

\begin{abstract}
An electron beam (e-beam) controlled switch makes use of the lowenergy secondary electrons in a diffuse $e$-beam sustained discharge as the conducting medium which carries the switched current. The conductivity of the gas in the switch is negligible before the $e$-beam is turned on because the circuit parameters are chosen so that the open circuit voltage which appears across the switch electrodes is well below the static breakdown voltage of the gas in the switch gap. The $e$-beam switch can interrupt direct current because the density of the electrons in the switch decays by recombination and attachment when the $e$-beam is turned off and the switch conductivity decreases. In typical circuits, this decay in conductivity causes the switch voltage to rise and the switch current to fall, i.e., the switch "turns off." This paper presents the results of a series of theoretical calculations which were performed in order to: 1) identify the electron transport and other gas properties which optimize the performance of $e$-beam switches, 2) evaluate the performance of several real gases for use in $e$-beam switches, and 3)
\end{abstract}

Manuscript received March 15, 1982; revised July 6, 1982. This work was supported in part by U.S. Air Force under Contract F3361578-C-2010.

The author is with the Westinghouse R\&D Center, Pittsburgh, PA 15235 . determine the laws which predict the scaling properties of $e$-beam switches. Results are presented for $\mathrm{N}_{2}, \mathrm{Ar}, \mathrm{a} \mathrm{N}_{2}: A r=1: 9$ mixture, and $\mathrm{CH}_{4}$. These results show that $\mathrm{CH}_{4}$ provides the best $e$-beam switch performance. Comparison of experimental results and theoretical predictions for $\mathrm{CH}_{4}$ supports both the theory and the predicted good e-beam switch performance for $\mathrm{CH}_{4}$.

\section{INTRODUCTION}

A N ELECTRON-BEAM ( $e$-beam) controlled switch makes use of the low-energy secondary electrons in a diffuse $e$-beam sustained discharge as the conducting medium which carries the switched current. The conductivity of the gas in the switch is negligible before the $e$-beam is turned on because the circuit parameters are chosen so that the open circuit voltage which appears across the switch electrodes is well below the static breakdown voltage of the gas in the switch gap. The $e$ beam switch can interrupt direct current because the density of the electrons in the switch decays by recombination and attachment when the $e$-beam is turned off and the switch con- 